03

\title{
Диагностика полей плотности фотометрическим теневым методом при гиперзвуковом обтекании конуса в легкогазовой баллистической установке
}

\author{
(C) П.П. Храмцов, В.А. Васецкий, В.М. Грищенко, М.В. Дорошко, М.Ю. Черник, А.И. Махнач, И.А. Ших
}

Институт тепло- и массообмена им. А.В. Лыкова НАН Беларуси, 220072 Минск, Беларусь

e-mail: iceret@list.ru

Поступило в Редакцию 14 февраля 2018 г.

В окончательной редакции 14 февраля 2018 г.

Принято к публикации 15 апреля 2019 г.

\begin{abstract}
Предложен новый способ получения гиперзвуковых течений и представлены результаты экспериментального исследования гиперзвукового обтекания конусов с углами полураствора $\tau_{1}=3^{\circ}$ и $\tau_{2}=12^{\circ}$ при числах Маха набегающего потока $M=18\left(\tau_{1}=3^{\circ}\right)$ и $14.4\left(\tau_{2}=12^{\circ}\right)$. Использование легкогазовой баллистической установки, в которой вместо канала разгона было установлено сопло Лаваля, позволило получить гиперзвуковой поток с высоким значением оптической плотности истекающего газа, достаточной для визуализации и диагностики течения оптическими методами. Визуализация картины течения производилась теневым методом ножа и щели. Теневые картины регистрировались с помощью высокоскоростной камеры Photron Fastcam с временем экспозиции $1 \mu$ s и скоростью съемки 300000 fps. Число Маха для набегающего потока рассчитывалось по теневым картинам на основе угла наклона ударной волны.
\end{abstract}

Ключевые слова: легкогазовая установка, гиперзвуковое течение, теневой метод, сопло Лаваля, число Maxa.

DOI: $10.21883 / J T F .2019 .10 .48165 .74-18$

\section{Введение}

Быстрое развитие современной авиации и космической техники привело к необходимости экспериментального и теоретического исследований стойкости различных материалов к воздействию твердых высокоскоростных частиц, а также задач обтекания тел гиперзвуковыми потоками. Для решения задач экспериментального моделирования высокоскоростного удара разработаны и успешно используются различные конструкции легкогазовых пушек, способных обеспечить разгон ударников сравнительно большой массы до скоростей, сопоставимых с первой космической скоростью. Условия проведения этих экспериментов (в частности, параметры рабочего газа) схожи с условиями исследования гиперзвуковых течений, поэтому определенные конструкции легкогазовых пушек при замене канала разгона на сопло Лаваля могут применяться для получения гиперзвуковых потоков с целью визуализации и диагностики гиперзвукового обтекания тел [1-4]. Начиная с 40-х годов прошлого века, получено множество экспериментальных данных, касающихся способов формирования, особенностей течения и методов исследования характеристик сверх- и гиперзвукового обтекания тел различных геометрий [5-16], а также накоплен большой теоретический материал [17-19]. С развитием вычислительной техники к экспериментальным способам диагностики гиперзвуковых потоков добавились методы численного моделирования [20-24]. Следует отметить, что экспериментальные исследования гиперзвукового обтекания объектов в силу важности решаемых задач являются предпочтительными. Для подтверждения и уточнения полученных результатов проводятся также полетные испытания, которые обеспечивают получение более надежных и достоверных данных. Число Маха определяется, как правило, путем измерения полного и статического давления в потоке при помощи насадка Прандтля либо используется расчетное число Маха для сопла Лаваля [5,6,10-12,14,15].

Метод измерения давления при использовании насадка Прандтля и метод измерения температуры с помощью термопар являются инвазивными, и их применение искажает реальную картину течения, а также усложняет конструкцию и монтаж исследуемых объектов вследствие необходимости размещения измерительных элементов внутри и на поверхности исследуемых моделей. В гиперзвуковых аэродинамических установках наиболее эффективными являются оптические методы исследования с использованием высокоскоростных камер для регистрации быстропротекающих процессов. Для получения картины течения в гиперзвуковых потоках используются методы лазерного ножа, дымовой визуализации, визуализации при помощи искрового разряда и т. д. [5,7,12,15]. Оптические методы, основанные на связи оптических и термодинамических свойств среды (например, теневые и интерференционные методы), позволяют проводить измерения в гиперзвуковом потоке бесконтактным способом с высокой точностью. В качестве метода, альтер- 
нативного термопарным измерениям, для визуализации и измерения поля температур на поверхности модели можно использовать термоиндикаторные покрытия [8,9].

При решении определенных задач современного авиастроения и ракетной техники иногда возникает необходимость совместного рассмотрения двух смежных вопросов: определения стойкости материалов к высокоскоростному удару и диагностики гиперзвукового обтекания тел. В связи с этими требованиями представляется целесообразным создание универсального испытательного стенда - компактного, быстродействующего и оснащенного оптической системой диагностики, на котором возможно проведение как баллистических испытаний, так и исследование картины течения и характеристик гиперзвукового обтекания объектов любой сложности.

\section{Экспериментальное оборудование и методика измерений}

Большинство экспериментальных схем, в которых реализуется гиперзвуковое истечение газа при больших значениях числа Маха, построено на основе ударной трубы. В торце трубы устанавливаются блок диафрагм и сопло Лаваля, через которое происходит истечение нагретого газа за фронтом отраженной ударной волны [25]. При этом вследствие высокой степени расширения газа в сопле Лаваля, рассчитанного на большое значение числа Маха, плотность результирующего потока оказывается достаточно низкой, что сильно затрудняет визуализацию потока оптическими методами. Значения углов

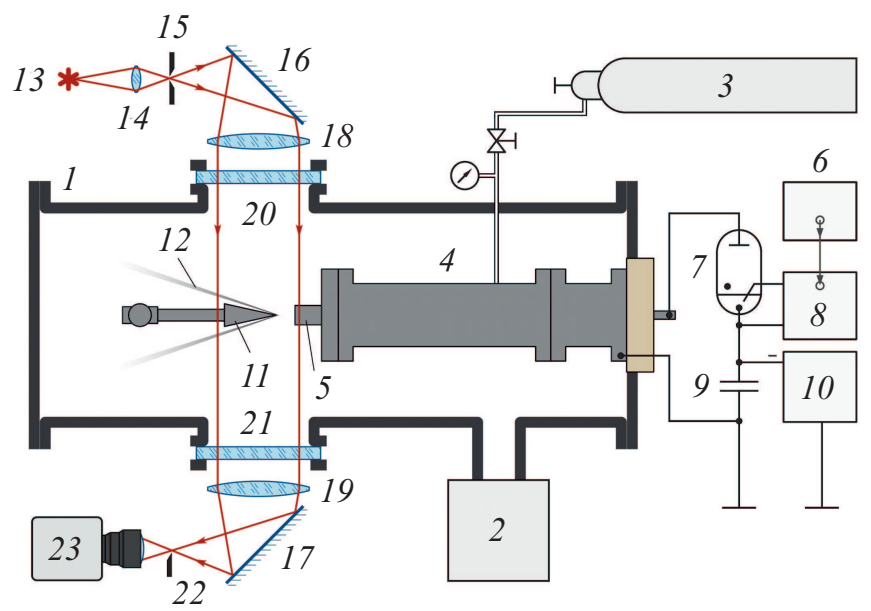

Pис. 1. Схема экспериментальной установки: 1 - вакуумная камера, 2 - вакуумный пост, 3 - баллон с гелием, 4 - легкогазовая секция, 5 - сопло, 6 - генератор импульсов, 7 - коммутирующее устройство (игнитрон), 8 - устройство запуска игнитрона, 9 - конденсаторная батарея, $10-$ высоковольтный источник напряжения, 11 - обдуваемая модель (конус), 12 - коническая ударная волна, 13 - источник света, 14 - конденсор, 15 - щель, 16,17 - поворотные зеркала, 18,19 - длиннофокусные объективы, 20,21 - оптические окна, 22 - нож Фуко, 23 - высокоскоростная камера.

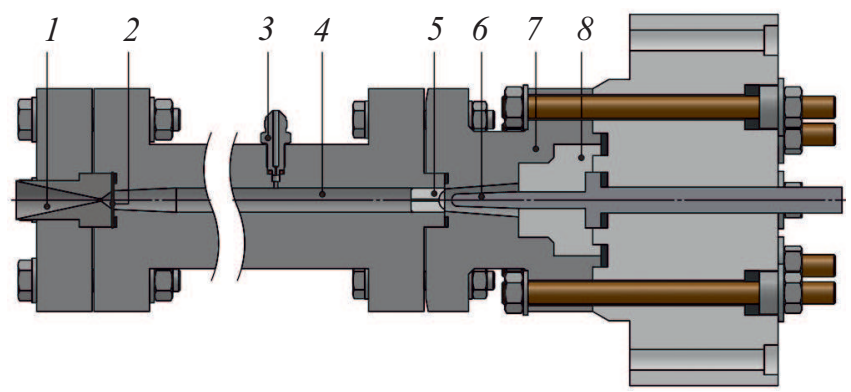

Рис. 2. Конструкция видоизмененного метательного устройства с соплом: 1 - сопло Лаваля, 2 - диафрагма, 3 - штуцер для напуска газа, 4 - канал, в котором происходит сжатие газа поршнем, 5 - поршень, 6 - центральный электрод (катод), 7 - анод плазменного ускорителя, 8 - изолятор.

отклонения зондирующего излучения при прохождении света через оптическую неоднородность определяются выражениями [26]:

$$
\begin{gathered}
\varepsilon_{x}=\int_{z_{1}}^{z_{2}} \frac{\partial \ln n(x, y, z)}{\partial x} d z, \\
\varepsilon_{y}=\int_{z_{1}}^{z_{2}} \frac{\partial \ln n(x, y, z)}{\partial y} d z,
\end{gathered}
$$

где $z_{1}$ и $z_{2}$ - координаты входа и выхода света из оптической неоднородности.

При этом градиенты показателя преломления газа $\partial n / \partial x$ и $\partial n / \partial y$ в случае истечения легкого газа в вакуум оказываются очень небольшими по величине. Например, в случае истечения гелия в вакуумную камеру с остаточным давлением 1 Torr при характерном масштабе обтекаемого образца $\sim 10^{-2} \mathrm{~m}$ величины градиентов показателя преломления не превышают $5 \cdot 10^{-6} \mathrm{~m}^{-1}$. Поэтому в ряде случаев для проведения диагностики таких течений приходится прибегать к использованию методов многоходовой интерферометрии, многолучевой интерферометрии, интерферометрии в поляризованном свете, явления аномальной дисперсии, а также голографических методов повышения чувствительности оптических измерений, что сильно усложняет методику и технику экспериментального исследования [27]. С другой стороны, использование легкогазовой баллистической установки, в которой вместо канала разгона с ударником установлено сопло Лаваля, позволяет получить гиперзвуковой поток с высоким значением оптической плотности истекающего газа и применить метод Теплера для его визуализации. Работа экспериментальной установки для гиперзвукового обдува в режиме баллистической установки подробно описана в [28].

Эксперименты по гиперзвуковому обдуву конусов проводились в вакуумной камере при остаточном давлении газа, равном 1 Torr. Схема экспериментальной установки представлена на рис. 1. 


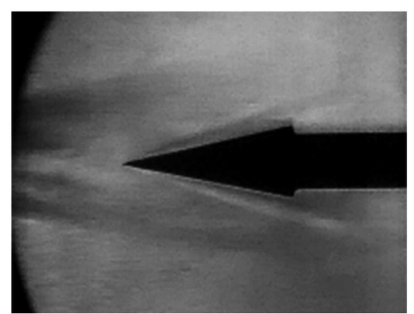

$125 \mu \mathrm{s}$

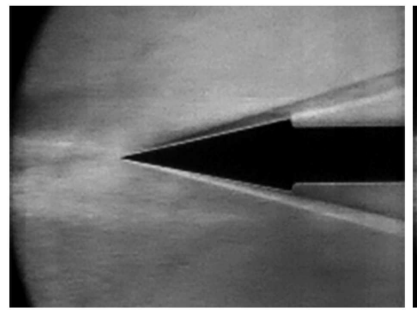

$1408 \mu \mathrm{s}$

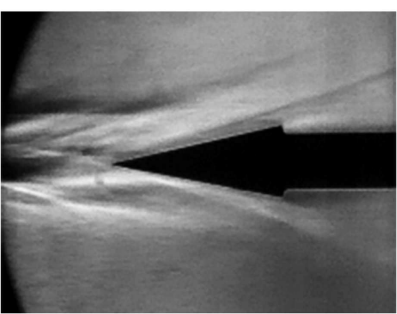

$208 \mu \mathrm{s}$

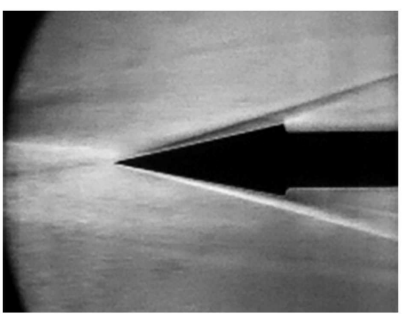

$1675 \mu \mathrm{s}$

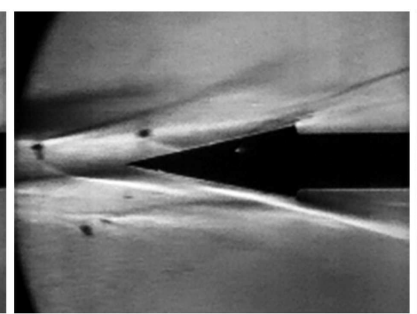

$258 \mu \mathrm{s}$

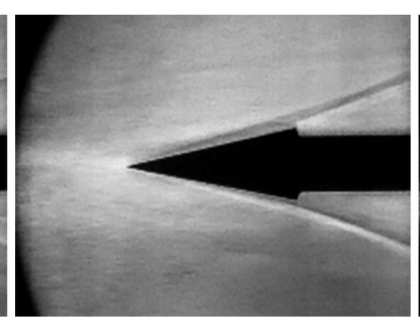

$2700 \mu \mathrm{s}$

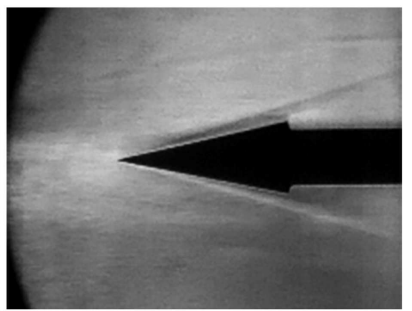

$675 \mu \mathrm{s}$

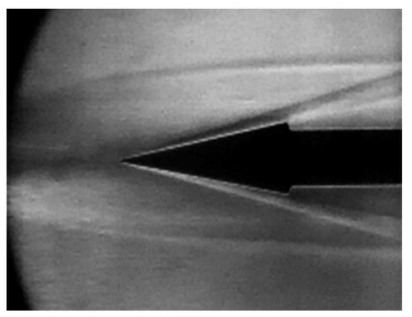

$3083 \mu \mathrm{s}$

Рис. 3. Скоростная сьемка гиперзвукового обтекания конуса с углом полураствора $\tau_{2}=12^{\circ}$.

Более детальная схема видоизмененного метательного устройства метательного устройства с соплом Лаваля приведена на рис. 2. В проведенных экспериментах легкогазовая секция заполнялась гелием до давления $~ 40$ bar. Поршень, вызывающий адиабатическое сжатие легкого газа, приводился в движение в результате работы эрозионного магнитоплазменного ускорителя. Конденсаторная батарея емкостью $1200 \mu \mathrm{F}$ заряжалась до напряжения 4-5 kV. Непосредственно перед конфузорной частью сопла Лаваля устанавливалась диафрагма, состоящая из 5 латунных мембран толщиной $100 \mu \mathrm{m}$ каждая. Разрыв диафрагмы происходил при давлении $\sim 1600$ bar. Степень сжатия и температуру рабочего газа можно оценить, исходя из уравнения Пуассона:

$$
\frac{T_{2}}{T_{1}}=\left(\frac{V_{1}}{V_{2}}\right)^{\gamma-1}=\left(\frac{P_{2}}{P_{1}}\right)^{(\gamma-1) / \gamma},
$$

где $P, V, T$ - давление, объем и температура газа соответственно, $\gamma$ - отношение удельных теплоемкостей для одноатомного газа, а индекс 1 соответствует состоянию газа до сжатия, индекс 2 - состоянию газа при разрыве диафрагмы.

Для представленных экспериментов степень сжатия рабочего газа в момент разрыва диафрагмы составляет $\sim 10$, а температура достигает $1500 \mathrm{~K}$. После разрыва диафрагмы продолжается дальнейшее сжатие газа при одновременном его истечении через критическое сечение сопла Лаваля. Максимальная степень сжатия, которая может быть рассчитана как отношение объема легкогазовой секции к объему конфузорной части сопла, для данной установки равна 50. Геометрические параметры сопла Лаваля для числа Маха $M=18$ рассчитывались по формуле [18]:

$$
\frac{A}{A^{*}}=\left(\frac{2}{\gamma+1}\right)^{\frac{\gamma+1}{2(\gamma-1)}} \frac{1}{M}\left(1+\frac{\gamma-1}{2} M^{2}\right)^{\frac{\gamma+1}{2(\gamma-1)}},
$$

где $A$ и $A^{*}$ - площади выходного и критического сечений сопла соответственно.

Обдуваемые модели конусов жестко фиксировались соосно с соплом на расстоянии $20 \mathrm{~mm}$ от его среза. Для визуализации картин гиперзвукового обтекания конуса использовался теневой метод ножа и щели. Ширина щели составляла $0.16 \mathrm{~mm}$, а нож Фуко устанавливался в фокальной плоскости приемной части теневого прибора таким образом, чтобы перекрывать половину изображения щели. В качестве источника света использовалась галогеновая лампа мощностью $150 \mathrm{~W}$. Фокусное расстояние теневого прибора составляло $1 \mathrm{~m}$. Регистрация теневых картин (рис. 3) проводилась с помощью высокоскоростной камеры Photron Fastcam с временем экспозиции $1 \mu \mathrm{s}$ и скоростью съемки $300000 \mathrm{fps}$.

\section{Результаты экспериментов и обсуждение}

Анализ результатов скоростной съемки теневых картин гиперзвукового обтекания конусов показывает, что в начальный промежуток времени, продолжительностью около 260-300 $\mu \mathrm{s}$, наблюдается поток, содержащий некоторое количество частиц, образовавшихся в результате разрушения диафрагмы, около каждой из которых виден свой конус Маха (рис. 3). В течение последующего интервала, равного $1.3 \mathrm{~ms}$, происходит нарастание скорости потока и выход сопла на стационарный режим. Продолжительность стационарного режима истечения из сопла на максимальной скорости достигает $4.5 \mathrm{~ms}$. 


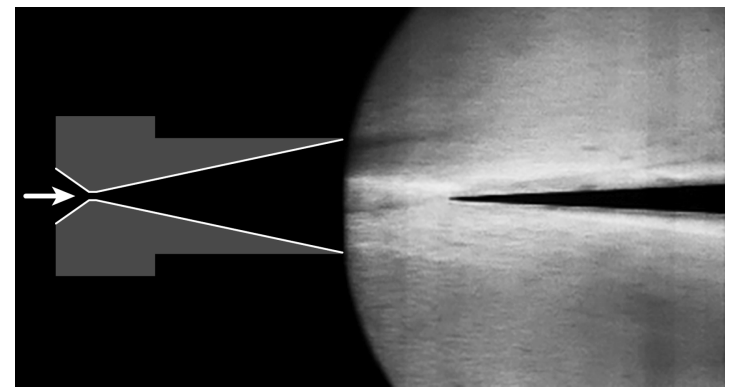

Рис. 4. Гиперзвуковое обтекание конуса с углом полураствора $\tau_{1}=3^{\circ}$.

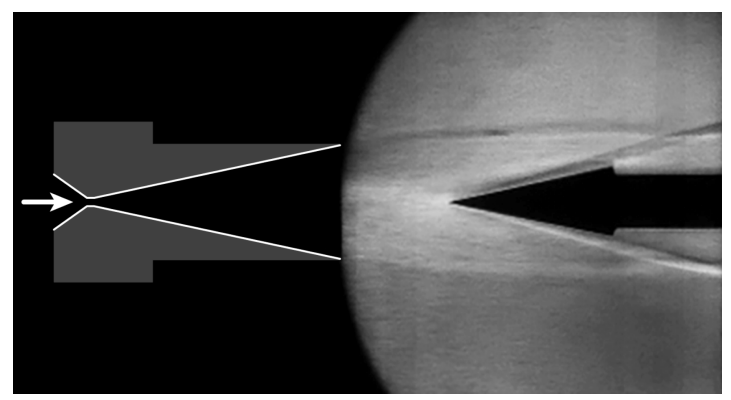

Рис. 5. Гиперзвуковое обтекание конуса с углом полураствора $\tau_{2}=12^{\circ}$.

При максимальной скорости набегающего потока на теневых картинах наблюдается наиболее острый угол наклона ударной волны. Далее по увеличению угла наклона ударной волны на теневых картинах можно судить о постепенном уменьшении скорости набегающего потока, что связано с падением давления в конфузорной части сопла.

На рис. 4 и 5 представлены теневые картины гиперзвукового обтекания конусов с углами полураствора $\tau_{1}=3^{\circ}$ и $\tau_{2}=12^{\circ}$, соответствующие моменту достижения максимального значения скорости набегающего потока.

Общая теория расчета сверхзвукового обтекания конуса позволяет определить характеристики течения на основе известного значения угла наклона $\sigma$ ударной волны $[17,18]$. Из этой теории получено аналитическое выражение для расчета чисел Маха при гиперзвуковой скорости обтекания [19]:

$$
M=\sqrt{\frac{2 \csc ^{2} \sigma\{C-\sec \sigma\}}{(\gamma+1)\left\{C+\frac{\cos \sigma}{\sin ^{2} \sigma}\right\}-(\gamma-1)\{C-\sec \sigma\}}},
$$

где $\tau$ - угол полураствора модели конуса, $M$ - число Маха набегающего потока, $C$ - константа, определяемая формулой

$$
C=\ln \left(\frac{\sin \sigma}{1-\cos \sigma}\right)-\ln \left(\frac{\sin \tau}{1-\cos \tau}\right)-\frac{\cos \tau}{\sin ^{2} \tau} .
$$

В данном исследовании угол наклона ударной волны измерялся по теневым картинам гиперзвукового обтекания моделей. В результате расчетов по формуле (4) получены значения $M_{1}=18$ для конуса с углом полураствора $\tau_{1}=3^{\circ}$ (рис. 4) и $M_{2}=14.4$ для конуса с углом полураствора $\tau_{2}=12^{\circ}$ (рис. 5).

\section{Анализ теневых картин и расчет полей плотности гелия при гиперзвуковом обтекании конуса}

Как известно из теории шлирен-метода [26], относительное изменение интенсивности света $\left(I-I_{0}\right) / I_{0}$ в приближении геометрической оптики прямо пропорционально углу отклонения луча $\varepsilon$ в оптической неоднородности и определяется соотношением

$$
\frac{I-I_{0}}{I_{0}}=\frac{\varepsilon f}{d}
$$

где $d=0.16 \mathrm{~mm}$ - ширина щели, $f=1000 \mathrm{~mm}-$ фокусное расстояние оптической системы, $I_{0}$ и $I-$ интенсивность света при отсутствии и наличии оптической неоднородности соответственно.

Поскольку обтекаемое тело обладает осевой симметрией, при обработке теневых картин использовалось допущение о наличии осесимметричной картины течения. Введем систему координат таким образом, чтобы начало координат совпадало с вершиной конуса, а оси располагались следующим образом: ось $x$ - нормально к кромке оптического ножа, ось $z$ - вдоль пучка проходящего света, а ось $y$ - вдоль оси симметрии конуса. В этом случае величины углов отклонения света связаны с распределением показателя преломления внутри изучаемой неоднородности посредством соотношений Абеля, получаемых из формулы (1) [26,29]:

$$
\varepsilon(x)=2 \int_{x}^{R} \frac{d \ln n(r)}{d r} \frac{x d r}{\sqrt{r^{2}-x^{2}}}
$$

где $x$ - текущая координата, $R$ - радиус исследуемой неоднородности.

В дальнейших расчетах использовалось обращение уравнения Абеля, в котором величина $d \ln n / d r$ представлена в явном виде:

$$
\frac{d \ln n(r)}{d r}=\frac{1}{\pi} \frac{d}{d r} \int_{r}^{R} \frac{\varepsilon(x) d x}{\sqrt{x^{2}-r^{2}}}
$$

После повторного интегрирования уравнения (7) получаем распределение показателя преломления по радиусу неоднородности [26,29]

$$
\ln \frac{n(r)}{n_{0}} \approx \frac{n(r)-n_{0}}{n_{0}}=\frac{\Delta n}{n_{0}}=\frac{1}{\pi} \int_{r}^{R} \frac{\varepsilon(x) d x}{\sqrt{x^{2}-r^{2}}},
$$

где $n_{0}$ - некоторое известное значение показателя преломления на линии интегрирования, например, в невозмущенной области течения $n_{0}=n(R) \approx 1$. 

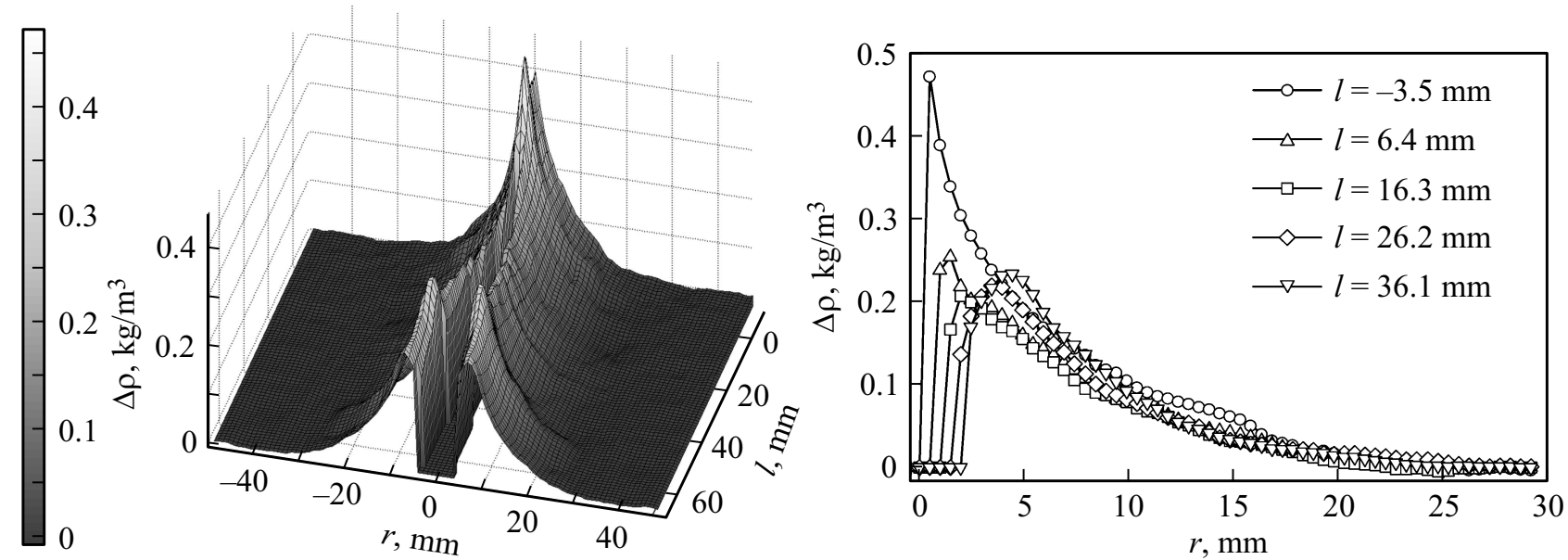

Рис. 6. Результаты расчета полей плотности гелия при гиперзвуковом обтекании конуса с углом полураствора $\tau_{1}=3^{\circ}$. Слева $3 D$-распределение поля плотности, справа - выделенные сечения.
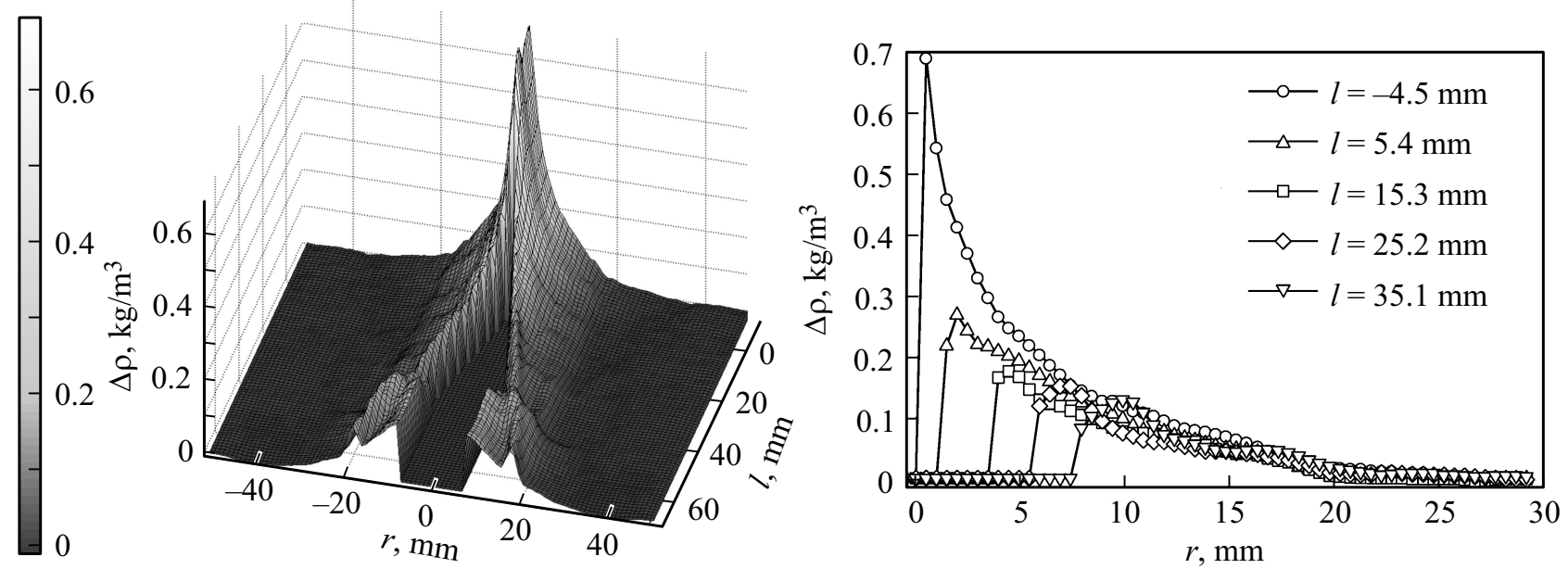

Рис. 7. Результаты расчета полей плотности гелия при гиперзвуковом обтекании конуса с углом полураствора $\tau_{2}=12^{\circ}$. Слева $3 D$-распределение поля плотности, справа - выделенные сечения.

Методика численного расчета показателя преломления по формуле (8) заключается в том, что исследуемая неоднородность разбивается на кольцевые зоны, число $N$ которых равно количеству пикселей изображения в направлении оси $x$. Величина угла отклонения $\varepsilon$ в пределах кольцевой зоны принимается постоянной. При этих условиях выражение (8) может быть представлено в виде суммы элементарных интегралов:

$$
\frac{\Delta n\left(r_{j}\right)}{n_{0}}=\frac{1}{\pi} \sum_{i=j}^{N-1} \varepsilon\left(r_{i}\right) \int_{r_{i}}^{r_{i}+1} \frac{d r}{\sqrt{r^{2}-r_{j}^{2}}} .
$$

Распределение показателя преломления в поле течения рассчитывается по формулам (5), (8) путем независимого интегрирования по каждому сечению, перпендикулярному оси симметрии конуса.

Величины показателя преломления в соседних сечениях, рассчитанные по этой методике, оказываются несвязанными между собой, что приводит к появлению характерных искажений в распределении показателя преломления. Эти искажения обусловлены высокой шумовой составляющей экспериментально полученной функции распределения интенсивности света $I$ вдоль оси $y$. Для устранения случайных шумов проводилось предварительное сглаживание этой функции в указанном выше направлении методом наименьших квадратов $[30,31]$ :

$$
\int_{y_{\min }}^{y_{\max }}\left(\frac{d^{2} I}{d y^{2}}\right)^{2} d y \rightarrow \min .
$$

При этом значения аппроксимирующей функции должны отличаться от значений исходной экспериментально полученной зависимости не более чем на величину, равную стандартному отклонению шумов интенсивности зондирующего излучения $I_{0}$ в области невозмущенного потока. 
Расчет плотности гелия осуществлялся с использованием соотношения Гладстона-Дейла $[26,29]$

$$
n-1=K_{\mathrm{He}} \rho,
$$

где $K_{\mathrm{He}}=0.19607 \mathrm{~cm}^{3} / \mathrm{g}$ - константа Гладстона-Дейла

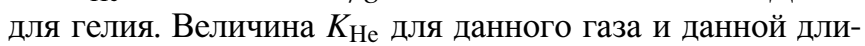
ны волны считается постоянной в большом диапазоне давлений. Ее значение было рассчитано, исходя из табличных значений плотности и показателя преломления газа при нормальных условиях [26]. Поскольку выбор начальных значений $n_{0}$ и $\rho_{0}$ является произвольным, на рис. 6,7 представлено распределение величины $\Delta \rho$, которая находится из соотношения

$$
\Delta n=K_{\mathrm{He}} \Delta \rho .
$$

Как можно видеть из рис. 6 и 7, плотность гелия в набегающем потоке претерпевает резкий скачок в зоне столкновения потока с поверхностью конуса и далее вниз по потоку наблюдается плавное снижение значения плотности по закону, близкому к экспоненциальному. Наличие малых флуктуаций плотности гелия на некотором расстоянии от острия конуса связано, по всей видимости, с потерей устойчивости в пограничном слое. Полученные данные о распределении плотности гелия в пограничном слое на поверхности конуса находятся в хорошем качественном согласии с результатами других авторов $[13,17,18]$.

\section{Заключение}

Модификация легкогазовой баллистической системы, в которой вместо канала разгона с ударником установлено сопло Лаваля, позволяет осуществить экспериментальное исследование обтекания моделей потоком, характеризующимся высокими значениями числа Маха и плотностью, достаточной для количественных оптических измерений теневыми или интерференционными методами.

\section{Конфликт интересов}

Авторы заявляют, что у них нет конфликта интересов.

\section{Список литературы}

[1] Smith F. // J. Fluid Mech. 1963. Vol. 17. N 1. P. 113-125. DOI: $10.1017 / \mathrm{S} 0022112063001154$

[2] Пилюгин Н.Н., Леонтьев Н.Е., Голубятников А.Н. // Успехи мех. 2003. Т. 2. Вып. 2. С. 97-124.

[3] Putzar R., Schaefer F. // Procedia Eng. 2015. Vol. 103. P. 421-426. DOI: 10.1016/j.proeng.2015.04.041

[4] Putzar R., Schaefer F. // Int. J. Impact Eng. 2016. Vol. 88. P. 118-124. DOI: $10.1016 /$ j.ijimpeng.2015.09.009

[5] Ridyard H.W. The aerodynamic characteristics of two series of lifting bodies at Mach number 6.86. NASA Research Memorandum NACA-RM-L54C15. 1954. 36 p.
[6] Rogers E.W.E., Berryand C.J., Davis B.M. Experiments with cones in low-density flows at Mach numbers near 2. A.R.C. Reports and Memoranda No. 3505. 1964. 44 p.

[7] Owens R.V. Aerodynamic characteristics of spherically blunted cones at Mach numbers from 0.5 to 5.0. NASA Research Note NASA-TN-D-3088. 1965. 60 p.

[8] Hubner J.P., Carroll B.F., Schanze K.S., Ji H.F., Holden M.S. // AIAA J. 2001. Vol. 39. N 4. P. 654-659. DOI: $10.2514 / 2.1358$

[9] Kurita M., Nakakita K., Mitsuo K., Watanabe S. // J. Aircr. 2006. Vol. 43. N 5. P. $1499-1505$. DOI: $10.2514 / 1.13608$

[10] Brosh A., Kussoy M.I. An experimental investigation of the impingement of a planar shock wave on an axisymmetric body at Mach 3. NASA Technical Memorandum NASA-TM84410. 1983. $165 \mathrm{p}$.

[11] Brown J.D., Brown J.L., Kussoy M.I. A documentation of twoand three-dimensional shock-separated turbulent boundary layers. NASA Technical Memorandum NASA-TM-101008. 1988. $105 \mathrm{p}$.

[12] Kussoy M.I., Horstmanand K.C. Intersecting shockwave/turbulent boundary-layer interactions at Mach 8.3. NASA Technical Memorandum NASA-TM-103909. 1992. $52 \mathrm{p}$.

[13] Петров К.П. Аэродинамика тел простейших форм. М.: Физматлит, 1998. 428 с.

[14] Радииг А.Н. Экспериментальная гидроаэромеханика. М.: МАИ, 2004. 296 c.

[15] Erdem E., Yang L., Kontis $K$. // 16th AIAA/DLR/DGLR International Space Planes and Hypersonic Systems and Technologies Conference. Bremen, Germany. 2009. P. 1-13. DOI: $10.2514 / 6.2009-7347$

[16] Saravanan S., Jagadeesh G., Reddy K.P.J. // Exp. Therm. Fluid Sci. 2009. Vol. 33. N 4. P. 782-790. DOI: $10.1016 /$ j.expthermflusci.2009.02.005

[17] Булах Б.М. Нелинейные конические течения газа. М.: Наука, 1970. 342 c.

[18] Лойщянский Л.Г. Механика жидкости и газа. М.: Дрофа, 2003. $840 \mathrm{c}$.

[19] Pottsepp L. // J. Aerosp. Sci. 1960. Vol. 27. N 7. P. 558-559. DOI: $10.2514 / 8.8642$

[20] Башкин В.А., Егоров И.В., Иванов Д.В., Пафнутьев В.В. // Ученые записки ЦАГИ. 2003. Т. XXXIV. Вып. № 3-4. C. 3-16.

[21] Коваленко В.В., Кравцов А.Н., Мельничук Т.Ю. // Ученые записки ЦАГИ. 2011. Т. ХLII. Вып. 1. С. 31-36. [Kovalenko V.V., Kravtsov A.N., Melnichuk T.Yu. // TsAGI Sci. J. 2011. Vol. 42. N 1. P. 37-46. DOI: $10.1615 /$ TsAGISciJ.v42.i1.30]

[22] Пилипенко А.А., Полевой О.Б., Приходько А.А. // Ученые записки ЦАГИ. 2012. Т. ХLIII. ВЫп. № 1. С. 3-31. [Pylypenko A.O., Polevoy O.B., Prykhodko O.A. // TsAGI Sci. J. 2012. Vol. 43. N 1. P. 1-36. DOI: $10.1615 /$ TsAGISciJ.2012005226]

[23] Галактионов А.Ю., Хлупнов А.И. // Вестник МГТУ им. Н.Э. Баумана. Сер. Машиностроение. 2015. № 5. C. 4-13.

[24] Димитриева Н.Ф. // Труды ИСП РАН. 2017. Т. 29. Вып. 1. C. 7-20.

[25] Гейдон А., Герл И. Ударная труба в химической физике высоких температур / Пер. с англ. М.: Мир, 1963. 428 c. [Gaydon A.G., Hurle I.R. The Shock Tube in High Temperature Chemical Physics. London: Verlag Chapman and Hall Ltd., 1963.] 
[26] Васильев Л.А. Теневые методы. М.: Наука, 1968. 400 с.

[27] Белозеров А.Ф. Оптические методы визуализации газовых потоков. Казань: КГТУ, 2007. 746 с.

[28] Khramtsov P.P., Vasetskiy V.A., Grishchenko V.M., Makhnach A.I., Chernik M.Yu., Shikh I.A. // High Temp. Mater. Processes. 2015. Vol. 9. N 3-4. P. 209-219.

DOI: 10.1615/HighTempMatProc.2016015794

[29] Скотников М.M. Теневые количественные методы в газовой динамике. М.: Наука, 1976. 160 с.

[30] Savitzky A., Golay M.J.E. // Anal. Chem. 1964. Vol. 36. N 8. P. 1627-1639. DOI: $10.1021 / \mathrm{ac} 60214 \mathrm{a} 047$

[31] Bockasten K. // J. Opt. Soc. Am. 1961. Vol. 51. N 9. P. $943-$ 947. DOI: $10.1364 / J O S A .51 .000943$ 